\title{
Reflets
}

Revue ontaroise d'intervention sociale et communautaire

\section{Le travail social auprès des familles}

\section{Michel-André Beauvolsk}

Volume 7, numéro 2, automne 2001

Le travail social en Ontario

URI : https://id.erudit.org/iderudit/026357ar

DOI : https://doi.org/10.7202/026357ar

Aller au sommaire du numéro

Éditeur(s)

Reflets : Revue ontaroise d'intervention sociale et communautaire

ISSN

1203-4576 (imprimé)

1712-8498 (numérique)

Découvrir la revue

Citer cet article

Beauvolsk, M.-A. (2001). Le travail social auprès des familles. Reflets, 7(2),

92-103. https://doi.org/10.7202/026357ar

Tous droits réservés (C) Reflets : Revue ontaroise d'intervention sociale et communautaire, 2001

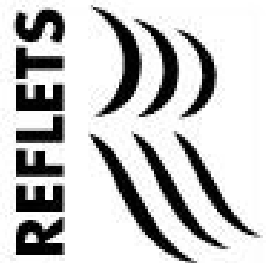

Ce document est protégé par la loi sur le droit d'auteur. L'utilisation des services d'Érudit (y compris la reproduction) est assujettie à sa politique d'utilisation que vous pouvez consulter en ligne.

https://apropos.erudit.org/fr/usagers/politique-dutilisation/ 


\section{Le travail social auprès des familles}

par

Michel-André Beauvolsk

École de service social, Université Laurentienne

Il est généralement admis que la famille est le système le plus important dans la vie de la majorité des personnes. Dans cet article, nous allons présenter un bref tableau du travail social auprès des familles. Nous allons d'abord tracer un historique des développements que nous considérons comme étant les plus importants puis, nous ferons une présentation des approches courantes. Nous terminerons par un résumé des questions et des défis soulevés par des praticiennes qui travaillent actuellement dans le domaine du travail social auprès de la famille.

\section{Historique}

La plupart des auteurs indiquent que le travail social auprès des familles remonte à la période de la Deuxième Guerre Mondiale (Goldenberg et Goldenberg 1985). En 1950, la pratique du service social auprès des enfants s'élargit pour inclure la dyade mèreenfant, s'inscrivant en cela dans la foulée des développements généraux dans ce domaine. Ce ne fut que tard dans les années 1950 ou au début des années 1960, que la famille, comme ensemble et totalité, est apparue dans les services sociaux et que les travailleuses sociales en intervention familiale en ont fait le groupe le plus important de leur pratique. 
Il est possible d'identifier cinq événements qui ont contribué au développement du travail social auprès des familles:

1) l'expansion du traitement basé sur la théorie psychanalytique,

2) l'introduction de la théorie des systèmes,

3) l'étude du rôle de la famille dans le développement de la schizophrénie chez un des membres de la famille (la thérapie familiale de la schizophrénie),

4) l'évolution du domaine du travail social auprès des enfants et,

5) l'intérêt dans les nouvelles approches telles que le travail auprès des petits groupes (Goldenberg et Goldenberg 1985).

Le traitement basé sur les théories psychanalytiques a permis et empêché à la fois l'expansion du travail social auprès des familles. Alors que Freud avait reconnu, dès le début, l'importance du rôle de la famille, notamment dans le processus d'identification chez l'individu, et, plus particulièrement, chez l'enfant, Bowen (1975) mentionne qu'à l'époque, plusieurs thérapeutes psychanalytiques pensaient que l'implication des autres membres de la famille aurait pour effet de contaminer le traitement du membre sous analyse.

En 1958, le psychanalyste Nathan Ackerman, considéré par plusieurs comme un des pères de la thérapie familiale, a adapté la théorie psychanalytique à son travail auprès des familles. Pour Ackerman, il existe une interaction constante entre l'individu biologique (un concept psychanalytique) et l'environnement social (un concept de la théorie des systèmes).Ainsi, d'après Ackerman, le fonctionnement d'une famille reposait sur le bon fonctionnement de chacun de ses membres dans leurs rôles respectifs et sur leur complémentarité, de telle sorte qu'un membre permette à l'autre de bien fonctionner et vice-versa. Dans ce cadre, les conflits familiaux peuvent apparaître à différents niveaux: chez un membre de la famille, entre ses différents membres, entre différentes générations de membres, entre la famille nucléaire et la famille étendue et, entre la famille et la communauté. Ainsi, un conflit peut créer une situation où les rôles arrêtent d'être complémentaires de telle sorte qu'un ou plusieurs membres développent des problèmes intrapsychiques. C'est ce qui arrive, par exemple, quand un membre devient le bouc-émissaire de la famille (Goldenberg et Goldenberg 1985). 
Les années 1950 et 1960 ont été le théâtre des développements les plus importants au niveau de la recherche et de la pratique du travail social auprès de la famille. En effet, c'est durant cette période que Ludwig von Bertalanffy (1956) formule sa théorie générale des systèmes. Il émet l'hypothèse que toute matière, qu'elle soit vivante ou non, possède des caractéristiques semblables et qu'elle interagit selon des règles précises. Peu de temps après, les praticiennes en santé mentale ont adopté une approche systémique et l'ont appliquée aux systèmes sociaux (Hearn 1969). À partir de ce moment, il est devenu pratiquement impossible de parler du travail social auprès de la famille sans s'appuyer sur la théorie des systèmes.

Vers la fin des années cinquante, Don Jackson (1960) met sur pied le Mental Research Institute (MRI) à Palo Alto, en Californie. Dans son groupe de recherche, on trouve, entre autres, Virginia Satir,Jay Haley, John Weakland, Paul Watzlawick et Richard Fisch, qui font figures de proue (Goldenberg et Goldenberg 1985). Ce sont ces théoriciens de Palo Alto qui ont développé la théorie de la communication en montrant qu'il était impossible de ne pas communiquer: «One cannot not communicate» (Watzlawick, Helmick Beavin et Jackson 1967: 61). Un des principes fondamentaux de la théorie de la communication affirme qu'un échange entre deux individus ne comprend pas seulement le contenu de la communication, mais aussi les messages implicites sur la nature de la relation existant entre les participants. Suite à cette découverte, les théoriciens de Palo Alto ont fait porter leurs recherches sur le lien entre la communication et la schizophrénie. C'est à partir de ce lien qu'il ont développé la théorie de la double contrainte (double bind theory). Celle-ci montre les effets destructeurs, sur un membre de la famille, de messages structurés, de telle sorte qu'une première affirmation se voit doubler d'une seconde affirmation qui contredit la première et que ces deux affirmations s'excluent mutuellement, ne permettant pas à l'individu d'identifier et de résoudre la contradiction (Nelsen 1986).

Virginia Satir, aussi membre du groupe de Palo Alto, a aussi travaillé sur la théorie de la communication, mais avec une perspective plus positive. En fait, on a appelé son approche le 
modèle humaniste (Goldenberg et Goldenberg 1985).Virginia Satir, travailleuse sociale en psychiatrie, a créé ce qui est probablement le premier programme formel de formation en thérapie familiale. Dans son livre, Conjoint Family Therapy (1964), elle a décrit la famille comme un système en équilibre comportant des «règles» et où chaque membre de la famille cherche à maintenir son estime de soi tout en concevant et en respectant que les autres membres ont besoin de faire la même chose. Une autre contribution de Satir est d'avoir identifié les rôles de conformiste (en anglais, placater), soit quelqu'un qui est toujours d'accord et ne s'affirme jamais; d'irresponsable (en anglais, blamer), soit un individu qui cherche toujours à blâmer les autres et, finalement, de personne négligeable (en anglais, irrelevant person), soit une personne qui ne compte pas. En fait, Satir a proposé que les gens qui ont une estime de soi faible utilisent ces rôles pour cacher leurs véritables sentiments. Finalement, Satir, dans son travail avec les familles, les aide à communiquer d'une façon claire et directe et à exprimer leurs vrais sentiments.

Après les années 1970, la personne reconnue pour son important apport à la pratique du service social auprès de la famille est le psychiatre, Salvador Minuchin. Né en Argentine, Minuchin a commencé à travailler en Israël avec les enfants victimes de l'holocauste. C'est en travaillant avec ces enfants qu'il a reconnu l'importance de la participation des familles au traitement. Minuchin a développé son approche structurelle à son arrivée aux États-Unis (Minuchin 1974). Dans son optique, l'organisation d'un système familial se comprend d'abord par ses structures. Dans une famille, les structures sont les règles invisibles qui déterminent son fonctionnement. Certaines règles sont communes à toutes les familles. De plus, il y a généralement une hiérarchie des pouvoirs; les parents, dans la plupart des cas, exercent une autorité sur les enfants. Minuchin monter que certaines fonctions sont complémentaires, comme les relations d'interdépendance entre les deux conjoints et que d'autres règles sont spécifiques à chaque famille et se développent après des années d'adaptation. Selon lui, les règles ont pour objectif le maintien d'un niveau acceptable et minimum de stabilité dans la famille. La structure qualifie le 
système et permet la résistance au changement. Chez certaines familles, ces structures peuvent nuire au fonctionnement des membres de la famille. L'objectif de l'approche structurelle est le rétablissement de structures permettant à la famille de bien fonctionner (Minuchin 1974).

Si Minuchin a imposé la pratique auprès des familles durant les années 1970, ce sont Haley (1976) et Madanès (1981), utilisant l'approche stratégique, qui ont dominé la pratique des années 1980. D'après eux, le rôle du thérapeute est d'identifier clairement les objectifs et de développer des stratégies pour résoudre les problèmes présentés par la famille. En général, dans cette approche, l'objectif principal est de régler les problèmes pour répondre aux besoins de la famille. Ce n'est pas la compréhension ou la prise de conscience qui va aider la famille, mais le processus de la résolution des problèmes entrepris par la famille en suivant les directives présentées par la thérapeute (Goldenberg et Goldenberg 1985).

Au cours des quinze dernières années, le travail social d'aide individuelle (casework) et son champ d'expertise a été défini, redéfini et raffiné pour tenir compte du rapport entre la personne et son environnement social. Étant donné que la famille est l'environnement social le plus important pour la croissance émotionnelle, sociale et intellectuelle de ses membres, il existe un passage quasi naturel, sinon obligé entre l'aide individuelle (casework) et l'intervention familiale. Ainsi, l'intervention familiale, comme modalité de traitement, a trouvé dans le travail social, un lieu propice à son développement, son expansion et son expérimentation. Afin de terminer cette revue de l'histoire, je présenterai, de façon particulière, certaines approches qui animent la pratique auprès des familles.

\section{Le modèle structural de Minuchin}

Populaire encore aujourd'hui, le modèle de Minuchin est clair et de compréhension facile. Quatre concepts essentiels constituent le fondement de l'approche structurale: la structure, les règles, les 
sous-systèmes et les frontières.Autour de ceux-ci, gravitent d'autres éléments qui complètent cette description de base. Les interactions familiales font partie des relations dont les séquences sont reconnues et même prévisibles. La structure familiale se décrit à travers les éléments suivants: le pouvoir partagé entre la mère et le père, les jeux d'alliance existant entre parents et enfants, les rôles rigides, un langage directif et une communication autoritaire. Les règlements font partie de deux systèmes de contraintes. Le premier régit la hiérarchie de pouvoir et le deuxième précise les attentes réciproques des membres de la famille. La hiérarchie représente l'organisation des différents niveaux de responsabilité du système familial. Les frontières ne sont autres que les règlements qui régissent la participation aux transactions familiales et leur mode opératoire. C'est ici, au niveau des frontières et de leur délimitation, que le rôle du travailleur social prend tout son sens, car il agit tel un véritable réparateur de frontières. Dans la famille désengagée où dominent des frontières interpersonnelles rigides, il cherchera à ouvrir et percer la ligne frontalière et, dans la famille enchevêtrée où dominent des frontières interpersonnelles diffuses, il cherchera à établir clairement la ligne frontalière (Elkaim 1995: 224).

\section{Le modèle narratif de Michael White}

C'est dans le contexte du constructivisme social qu'on peut comprendre une des affirmations de White selon laquelle l'attention doit porter sur l'objectivation du problème plutôt que sur la personne. De ce point de vue, ce n'est pas la dynamique personnelle ou familiale qui explique le problème, mais la dynamique du problème qui organise les réactions de la personne et de la famille (Elkaim 1995: 538). C'est en posant au client plusieurs questions que les problèmes sont extériorisés. De cette façon, les clients parviennent à repenser leur relation en fonction de leur histoire de vie, en se rendant compte combien elle les opprime. Ils se mobilisent alors contre elle et font finalement l'apprentissage de leur propre pouvoir en devenant de plus en 
plus conscients de l'influence qu'ils sont capables d'exercer. Il s'ensuit un travail de déconstruction.

Les deux aspects les plus importants de cette approche sont la métaphore narrative elle-même et la technique de l'extériorisation des problèmes. Ces deux idées représentent un renversement radical de la thérapie familiale traditionnelle. Mais, la force et la faiblesse de l'approche narrative est qu'elle fait appel uniquement à la dimension cognitive. Elle utilise des techniques de questionnement pour parvenir à la déconstruction de l'histoire, à l'ouverture d'espace sur d'autres histoires, à l'expression de préférence, de style de développement, de signification et d'extension de l'histoire à venir. Ainsi en extériorisant les problèmes et en déconstruisant d'une manière rigide les croyances culturelles et en démontrant une confiance dans les habiletés des clients à créer la meilleure recette de changement pour eux, les thérapeutes de ce modèle ont fait avancer la thérapie familiale (Elkaim 1995).

\section{Le modèle centré sur les solutions}

La thérapie centrée sur la solution est un modèle qui est apparu au cours de la dernière décennie. De Shazer et son équipe ont intégré les idées du constructivisme social dans un modèle beaucoup plus pragmatique. Les thérapeutes de ce modèle ne sont pas intéressés à identifier et à réactiver les discours narratifs culturels. Ils ne cherchent qu'à aider les gens à décentrer leur attention de leurs problèmes et à se concentrer sur les solutions possibles.

Le but de cette approche est d'amener les clients à comprendre et à analyser leurs problèmes dans un autre langage, centré sur ce qui aide ou ce qui pourrait aider dans l'avenir. Le travail des thérapeutes consiste à amener leurs clients à formuler des solutions. Les thérapeutes soutiennent la démarche du client en qui ils font entièrement confiance pour trouver la meilleure solution afin de résoudre la situation problématique. Ils ne cherchent pas à imposer une solution particulière. Au contraire, ils adhèrent à une vision 
orientée vers les forces, se concentrant sur les ressources et les succès personnels des personnes (Elkaim 1995).

\section{La thérapie symbolique-expérientielle}

Ce modèle de thérapie familiale a pour objet l'intégration de deux mondes: celui de l'expérience et celui des symboles. La tâche du thérapeute consiste à conduire la famille à vivre des expériences qui puissent modifier certains symboles dysfonctionnels. La participation de la famille et son engagement dans le traitement permettent d'engendrer de nouvelles informations qui, répercutées au niveau des processus non rationnels qui régissent son inconscient, entrainent la modification des symboles autour desquels une réorganisation devient alors possible. Whitaker et Keith (1982) ont défini quatre étapes dans le processus thérapeutique: la bataille pour la structure, la bataille pour l'initiative, l'épreuve du travail et la fin de la thérapie. Le processus devient souvent un jeu ou une danse où le thérapeute agit comme agent catalyseur des possibilités créatives de la famille. En terminant, il importe de se rappeler que les postulats théoriques de cette approche ne sont toujours très clairs mais, seule l'expérience peut l'apprendre (Elkaim, 1995).

\section{Les défis actuels}

Le travail social auprès de la famille se pratique dans des agences publiques, comme les Centres de l'enfant et de la famille ou dans des agences privées, comme le Service familial. Pourtant, ce champ n'est pas réservé aux seuls travailleurs sociaux, des psychologues et des éducateurs offrent aussi de l'intervention.

Le niveau de formation varie, allant d'une formation de base à des études supérieures de niveau maîtrise. Au plan commu- 
nautaire, de nombreux services offrent le travail social auprès de la famille tels que le programme de traitement affirmatif du Centre de santé mentale du Nord-Est de l'Ontario, anciennement connu sous le nom de Network North.

Selon Dianne Beska et Bev Shinke, deux praticiennes du Service familial d'Ottawa-Carleton travaillant actuellement auprès des familles, il y a eu des changements dans la famille moderne et, ceux-ci posent d'importants défis aux praticiennes. Le changement de clientèle est le plus remarquable. Par exemple, on est passé d'une clientèle dominé par la famille nucléaire traditionnelle occidentale à des familles immigrantes provenant de pays en voie de développement. Ces familles sont habituellement constituées de réfugiés échappant à des conflits armés ou des guerres, à la famine ou à d'autres désastres humains. Ces familles arrivent avec leurs cultures, leurs religions et leurs traditions souvent très différentes de pays occidentaux comme le nôtre. Si ces familles ont des enfants, les enfants seront élevés dans une culture très différente de celle des parents, ce qui aura l'effet de créer des conflits entre les générations.

Dans son livre intitulé Pour une intervention sociale efficace en milieu interculturel, Mvilongo-Tsala (2001), basant sa réflexion sur le double concept de centration et décentration culturelle, insiste qu'une décentration culturelle est nécessaire pour que les praticiennes et praticiens en intervention sociale puissent comprendre et surtout aider leurs clients. L'auteur réfere à une démarche paradoxale où la personne est invitée, en premier lieu, à reconnaitre les traditions et les valeurs de sa propre culture pour ensuite s'ouvrir à la culture de l'autre, en second lieu. L'interculture ainsi atteinte est de plus en plus présente dans nos communautés et l'identité culturelle doit inclure parfois une identité d'adoption, c'est-à-dire la reconnaissance de la richesse culturelle de l'autre.

Un autre changement est la croissance du nombre des familles recomposées. La Loi sur le divorce sans égard à la faute (1985) a changé radicalement l'institution du mariage. Si dans les années 1990, plus de $50 \%$ des mariages se terminaient par une rupture, la popularité de l'union libre et la distance prise par beaucoup de 
couples face à l'institution maritale font que les familles recomposées augmentent en nombre. Cette forme de familles amène avec elles de nouveaux défis d'adaptation, de loyauté et de permanence (Beauvolsk 1995).

Un troisième changement est l'augmentation du nombre de couples formés d'homosexuels ou de lesbiennes. Ces couples, qui veulent être acceptés dans un monde qui valorise toujours le couple hétérosexuel, ont des besoins et rencontrent des défis particuliers. Dans les dernières années, beaucoup a été écrit sur le sujet des couples de lesbiennes (Martindale 1998). Un couple lesbien doit faire face à plusieurs défis, par exemple, d'être accepté dans le rôle comme parent, surtout si l'enfant est un garçon. Travailler avec les couples d'homosexuels ou de lesbiennes risque de menacer les valeurs de base de la praticienne.

En plus des changements dans la famille, deux thèmes se présentent souvent dans les sessions d'intervention avec les familles. D'abord, il y a la question de la violence. Au cours des dernières années, on a commencé à développer un outil d'évaluation du degré de violence qui existe chez les familles (Edelson 1999; Whitney et Davis 1999). Beaucoup de protocoles d'évaluation obligent l'intervenante à soulever la question de l'existence ou non de la violence dans l'histoire de la famille. S'il y en a eu, il y a des règles à suivre afin d'assurer la protection de la victime avant même de commencer à travailler sur la dynamique de la famille. En outre, Bev Shinke rencontre de plus en plus de parents qui sont à bout de discipliner leurs adolescents. Elle trouve qu'il y a eu une augmentation au niveau de l'agressivité chez les adolescents. Les parents se plaignent que leurs adolescents n'acceptent aucun contrôle. Peut-être est-ce en raison du fait que les parents doivent travailler des longues heures à l'extérieur de la maison et que les familles ont moins de temps familial? Toujours est-il que Shinke passe beaucoup de temps à négocier des compromis entres les parents et leurs adolescents pour permettre à la famille de vivre ensemble. Elle trouve que la profession doit intervenir le plus rapidement possible pour établir des frontières (règles) et aider la famille à mieux fonctionner comme système. 
Un autre thème est le partage des tâches. Loin d'être nouveau comme thème, la femme continue, dans la majorité des familles, à être responsable de la plus grande partie des tâches domestiques. Pour aider une famille à s'entendre sur un partage de tâches équitable, l'intervenante doit confronter les traditions, les stéréotypes qui sont difficiles à changer.

\section{Conclusion}

La famille est un système et à ce titre, évolue dans un ensemble qui comprend d'autres systèmes. Lorsque des situations problèmes se présentent, elles peuvent provenir du système familial, des systèmes environnants ou de l'interaction entre le système familial et les autres systèmes. De plus, la personne est un système complexe. Il est donc illusoire de croire que le simple fait de travailler avec la famille lui permettra de résoudre toutes ses difficultés. Il arrive souvent qu'il faille intervenir à plusieurs niveaux en même temps, soit avec la famille comme système ou avec les personnes comme individus dans ce système. Cela dit, il n'en demeure pas moins que l'approche familiale offre une aide substantielle aux familles qui sont aux prises avec des difficultés d'interaction. La recherche sur l'efficacité de l'intervention familiale, bien qu'elle soit limitée, semble confirmer le truisme selon lequel l'intervention familiale réussit mieux lorsqu'elle est appliquée aux problèmes entre parents et enfants, aux problèmes familiaux et matrimoniaux.

\section{Bibliographie}

ABELSOHN, D. et D. GRAHAMS, D. (1991). «Adolescent Adjustment to Parental Divorce: An investigation from the Perspective of Basic Dimensions of Structural Family Therapy», Family Process, vol. 30, no 2, 177-190.

ACKERMAN, N.W. (1958). The Psychodynamics of Family Life, New York, Basic Books.

BARKELY, R. A., D.C. GUEREMONT et al. (1960). "A Comparison of Three Family Therapy Programs for Treating Family Conflicts in Adolescent with Attention-Deficit Hyperactivity Disorder», Journal of Consulting and Clinical Psychology, vol. 3, 450-462. 
BEAUVOLSK, M. A. (1995). «Le phénomène d'ajustement de la famille reconstituée francoontarienne», dans C. Bernier, S. Larocque et M.Aumond, M. (dir. de publ.), Familles Francophones, Multiples Réalités. Sudbury, Institut Franco-Ontarien, Collection Feuille de Trille, 181-194.

BERTALANFFY, L.V. (1968). General Systems Theory, New-York, Braziller.

EDELSON, J. L. (1999). «The Overlap between Child Maltreatment and Women battering», Violence Against Women, vol. 5, no 1,18-24.

ELKAIM, M. (1995). Panorama des thérapies familiales, Paris, Seuil.

FIGLEY, C. R., et T. NELSON (1990). «Basic Family Therapy Skills II: Structural Family Therapy», Journal of Marital and Family Therapy, vol. 16, no 3, 225-239.

FRIEDLANDER, M. L. et J. WILDMAN (1991). «Interpersonal Control in Structural and Milan Systemic Family Therapy", Journal of Marital and Family Therapy, vol. 17, no 4, 395-408.

GOLDENBERG, I. Et H. GOLDENBERG (1985). Family Therapy: an Overview (2ème edition), Pacific Grove, California, Brooks/Cole Publishing Company.

GREENBERG, A. et F et G.J. NEIMEYER (1986). «The Impact of Structural Family Therapy Training on Conceptual and Executive Skills», Family Process, vol. 25, no 4, 599-608.

HALEY, J. (1976). Problem-Solving Therapy, San Francisco, Jossey-Bass.

HEARN, G. (1969). «General Systems Theory and Social Work», dans F. Turner (dir.) Social Work Treatment, New York, Free Press, 333-359.

MADANES, C. (1981). Strategic Family Therapy, San Francisco, Jossey-Bass.

MARTINDALE, K. (1998). «What makes lesbianism thinkable? Theorizing lesbianism from Adrienne Rich to QueerTheory», dans N. Mandell (dir.), Feminist Issues: Race, class, and sexuality, Scarborough, Ontario, Prentice-Hall/Allyn and Bacon Canada, 42-51.

MINUCHIN, S. (1974). Families and family therapy, Cambridge, Massachusetts, Harvard University Press.

MVILONGO-TSALA, A. (2001). Pour une intervention sociale efficace en milieu interculturel, Paris, L'Harmattan.

NELSEN, J. (1986). «Communication Theory and social Work Treatment», dans F. Turner (dir. de publ.), Social Work Treatment, New York, Free Press, 219-243.

SATIR,V. (1964). Conjoint Family Therapy, Palo Alto, California, Science and Behavior Books.

WATZLAWICK, P., J. HELMICK BEAVIN et D. JACKSON, D. (1967). Pragmatics of Human Communication: A Study of Interactional Patterns, Pathologies and Paradoxes, New York, Norton.

WHITNEY, P., DAVIS, L. (1999). «Child abuse and domestic violence in Massachusetts: Can practice be integrated in a public child welfare setting?», Child Maltreatment, vol. 4, no 1, 98-112. 